1 Department of Oral Medicine, Faculdade de Odontologia, Universidade de São Paulo (São Paulo/Brazil).
Corresponding author:

Wladimir Gushiken de Campos School of Dentistry (Stomatology Department), University of São Paulo 2227. Professor Lineu Prestes Avenue, São Paulo/São Paulo, Brazil. ZipCode: 05508-000 Tel.: +55 11 3091-7883 E-mail: wgushiken@hotmail.com

Received: June 07, 2018

Accepted: November 09, 2018

\section{Does type 2 diabetic osteoporotic patients present more periodontal risks than non-osteoporotic patients? An evaluation with mandibular cortical index (Klemetti)}

Wladimir Gushiken de Campos' ${ }^{1}$, Gonzalo André Montesinos ${ }^{1}$, Rosa Cristina Peinado Agudo', Kaisermann Costa $^{1}$, Luciana Munhoz ${ }^{1}$, Emiko Saito Arita ${ }^{1}$
Aim: This study aim was to evaluate if patients with type 2 diabetes and osteoporosis have an increased risk of periodontal disease (horizontal and vertical bone loss) when compared to diabetic patients without osteoporosis. Additionally, to assess if patients with diabetes and osteoporosis have a greater risk of reduction of bone mineral density in the mandible, expressed by mandibular cortical index $(\mathrm{MCl})$ when compared to diabetic patients without osteoporosis. Methods: 59 patients (39 diagnosed with type 2 diabetes and osteoporosis; 20 diagnosed with type 2 diabetes and without osteoporosis) were selected. Type 2 diabetes was previously diagnosed by glycated hemoglobin examination and osteoporosis by peripheral dual-energy x-ray absorptiometry. Mandibular cortical index, as well as the presence of vertical and horizontal bone loss was verified on panoramic radiographs. Adjusted odds ratio analyses were performed on presence of periodontal disease and $\mathrm{MCl}$ considering the effect of osteoporosis. Results: Absence of statistical significance between variables was found. Conclusions: There is no difference between the risk of periodontal disease or low $\mathrm{MCl}$ among osteoporotic and non-osteoporotic type 2 diabetic patients.

Keywords: Osteoporosis. Diabetes mellitus, type 2. Alveolar bone loss. Periodontitis. 


\section{Introduction}

Osteoporosis is a chronic osteometabolic disease, with multiple influencing determinants, such as ageing and hormonal factors, which presents systemic reduction of bone mineral density (BMD) and bone microstructural alterations (BMA). Type 2 diabetes is a metabolic disease that affects the quality of bone trabeculae, due to the disturbance of bone material properties related to collagen matrix ${ }^{1}$.

Type 2 diabetes has also influencing factors, which worsen disease severity, such as obesity, dyslipidemia and periodontal disease ${ }^{2}$. There is a complex relationship between diabetes and periodontal disease ${ }^{3}$. Additionally, there is an association of periodontal disease and osteoporosis, which is widely studied in literature and results are contradictory ${ }^{4}$. However, results point that osteoporosis increases the risk of periodontal disease development ${ }^{4}$.

Periodontal disease is a chronic inflammatory disease highly prevalent in adulthood population, which leads to loss of periodontium integrity and may result in alveolar bone loss and, consequently, teeth loss 5 .

The diagnosis of osteoporosis is performed by dual x-ray absorptiometry (DXA), which is the main examination to detect changes in BMD. Notwithstanding, DXA is not highly available in developing countries. Therefore, a number of screening tools have been developed to screen patients at risk of low BMD, using low cost examinations, such as digital panoramic radiographs ${ }^{6}$.

Panoramic radiographic examination is often applied at the beginning of dental clinical treatment to new patient's evaluations, as suggest by the American Dental Association. Panoramic radiographs provides information on the maxillofacial area and adjacent structures with low radiation exposure ${ }^{7,8}$, and has great patient tolerance. Panoramic radiographs can also be applied as a screening tool to low BMD risk, when radiomorphometric indices are evaluated ${ }^{9}$.

Among the radiomorphometric indices that can be assessed on panoramic radiographs, the $\mathrm{MCl}$ is advocated to be inversely correlated with systemic BMD? ${ }^{9}$ The $\mathrm{MCl}$ evaluates the appearance of the cortical bone at the mandibular endosteal inferior margin of the mandible $e^{9}$, from the mental foramen to the posterior limit of the mandibular angle. The mandibular endosteal margin was classified as: $\mathrm{C} 1=$ normal, C2 = moderately; and C3 = severely eroded ${ }^{6,9}$.

Thus, the primary objective of this study was to evaluate if patients with type 2 diabetes and osteoporosis have an increased risk of periodontal disease (horizontal and vertical bone loss) when compared to diabetic patients without osteoporosis. Additionally, verify if patients with diabetes and osteoporosis have a greater risk of reduction of bone mineral density in the mandible, expressed by $\mathrm{MCl}$ when compared to diabetic patients without osteoporosis.

\section{Materials and methods}

\section{Study participants and inclusion and exclusion criteria}

This investigation was conducted with 59 type 2 diabetes patients who underwent concomitant panoramic radiographic and forearm dual-energy $\mathrm{x}$-ray absorptiometry 
(for screening osteoporosis) examination. All patients willing to participate in this study signed an informed consent form, and approval was obtained from the Ethics Committee of São Paulo University-Dentistry School (number FR 358902); protocol 145/10). The guidelines of Helsinki were followed in this study.

Selected participants filled out a form with personal information, medical history and lifestyle habits. Type 2 diabetes was previously diagnosed by glycated hemoglobin examination (values of glycated hemoglobin $>6,5 \%)^{10}$ and osteoporosis by peripheral dual-energy $x$-ray absorptiometry.

The 59 diabetic patients were divided into two groups: 39 with osteoporosis and 20 without osteoporosis, considering World Health Organization criteria ${ }^{11}$. Patients with T-scores values under -2.5SD were considered osteoporotic ${ }^{6,11}$.

Panoramic radiographic examinations with technical failures were excluded. Patients with other osteometabolic diseases or medications that affects bone metabolism; tobacco or alcohol chronic use; with less than 14 teeth and clinically confirmed aggressive periodontal disease were also excluded.

\section{Dual X-ray absorptiometry}

Forearm bone densitometry measurements were conducted with peripheral dual energy X-ray absorptiometry (pDEXA, Norland, Norland Medical Systems, Inc., White Plains, NY, USA. The scanning resolution was $1.00 \mathrm{~mm} \times 1.00 \mathrm{~mm}$ ); the forearm area selected for bone mineral density evaluation was the proximal radius.

\section{$\mathrm{MCl}$ and presence of periodontal bone loss assessment}

All digital panoramic radiographic images were taken using the same device (Kodak 8000, Eastman Kodak Company, Rochester, USA) and were processed on the same software (ImageJ, National Institute of Health, Bethesda, MD, USA).

Presence of periodontal bone loss was classified as "horizontal" or "vertical" according to the appearance of alveolar bone of the remaining teeth exclusively in panoramic radiographs.

The $\mathrm{MCl}$ was assessed by assessing the appearance of the cortical bone at the mandibular endosteal margin on both sides of the mandible, from an anterior limit below the mental foramen to the posterior limit at the mandibular angle, using a previously described criteria by Klemetti et $\mathrm{al}^{9}$. The mandibular endosteal margin was classified as: $\mathrm{C} 1$ = normal, when presenting sharp and marked continuous endosteal margin; $\mathrm{C}_{2}$ = moderately eroded, when presenting evidence of lacunar resorption; and C3 = severely eroded, with evident reduction of mandibular bone mineral density. Figure 1 illustrates the aforementioned classification.

All panoramic radiomorphometric assessment was accomplished independently in a random order by two $\mathrm{MCl}$ trained observers (dentists with expertise in oral radiology). Intraobserver reliability was assessed between measurements performed one week apart to eliminate memory bias. 

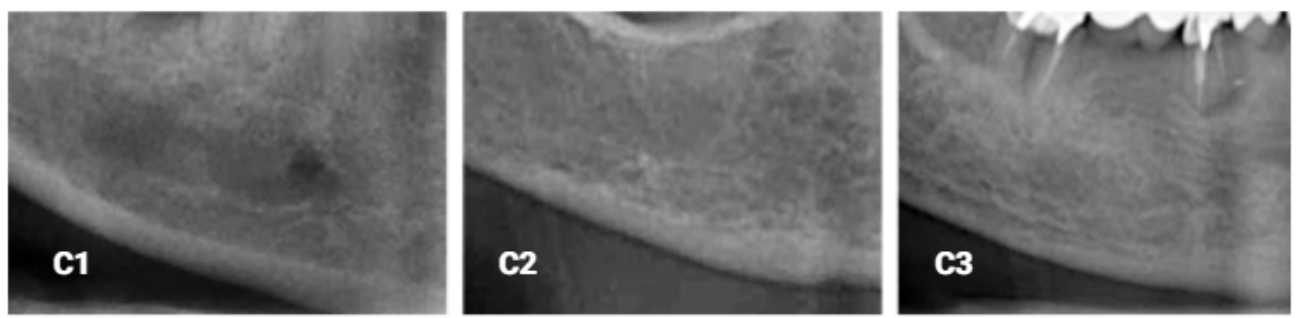

Figure 1. Examples of mandibular cortical index classification in panoramic radiographs (detail of mandibular cortex).

Table 1. Groups demographic characteristics. Median proximal radius T-score, median age, gender and median $\mathrm{MCl}$ of the participants evaluated.

\begin{tabular}{|c|c|c|c|c|c|}
\hline \multirow{2}{*}{ Group } & \multirow{2}{*}{ T-score } & \multirow{2}{*}{ Age } & \multicolumn{2}{|c|}{ Gender } & \multirow{2}{*}{ Median $\mathrm{MCl}$} \\
\hline & & & Male & Female & \\
\hline Diabetic and osteoporotic & -3.33 IR 1.15 & 65 IR 2 & 11 & 28 & 2 IR 2.6 \\
\hline $\begin{array}{l}\text { Diabetic and } \\
\text { non-osteoporotic }\end{array}$ & -1.26 IR 1.14 & 65 IR3 & 9 & 11 & 2 IR 1.1 \\
\hline
\end{tabular}

Abbreviations: IR: interquartile range; $\mathrm{MCl}$ : mandibular cortical index

\section{Statistical Analysis}

As a convenience sample was used for this study, no calculation of sample size was applied. Normality was assessed for variables using the Shapiro-Wilk test ${ }^{12}$.

Conditional logistic regression was used to assess the association between vertical and horizontal periodontal bone loss, $\mathrm{MCl}$ and osteoporosis. DXA results were stratified using the cut-off point value of $-2.5 \mathrm{SD}$. For $\mathrm{MCl}$, the " $\mathrm{C} 1$ " classification was selected as the cut-off point, as represents absence of alterations in mandibular cortex. "C2" and "C3" classifications were selected as low $\mathrm{MCl}$. Conditional logistic regression was used to assess the association between $\mathrm{MCl}$ and ethnicity. Risk estimates were presented as ORs with $95 \%$ Cls.

Intra- and inter-observer agreements were assessed using the Kappa test for $\mathrm{MCl}$.

All statistical analyses were performed using the IBM SPSS Statistics 24 software (SPSS, Inc., Chicago, IL, USA), with a significance level of $p<0.05$.

\section{Results}

\section{Descriptive statistics}

A total of 59 type 2 diabetes patients were evaluated: 39 osteoporotic and 20 non-osteoporotic. Median T-scores of the diabetic and osteoporotic patients was -3.33 IR and diabetic non-osteoporotic patients was $-1.26 \mathrm{IR}$. Median age was 65 years old for both groups. Results of descriptive statistics were presented in Table 1.

The group of diabetic and osteoporotic patients was composed of 11 men and 28 women. The group of diabetic and non-osteoporotic was composed of 9 men and 11 women. 
Table 2. Logistic regression results

\begin{tabular}{lcc}
\hline Variables & AOR $(95 \% \mathrm{Cl})$ & $\mathrm{p}^{*}$ \\
\hline $\begin{array}{l}\text { Horizontal bone loss } \\
\text { Absence }\end{array}$ & 1 & 0.740 \\
Presence & $0.795(0.205-3.082)$ & \\
\hline $\begin{array}{l}\text { Vertical bone loss } \\
\text { Absence }\end{array}$ & 1 & 0.601 \\
Presence & $0.670(0.150-3.005)$ & \\
\hline MCl & 1 & 0.314 \\
Normal & $1.815(0.569-5.791)$ & \\
Low &
\end{tabular}

Abbreviations: AOR: Adjusted Odds Ratio; Cl: Confidence Interval; MCl: Mandibular cortical index.

\section{Intra and interobserver test for $\mathrm{MCl}$}

Intraobserver agreement for $\mathrm{MCl}$ was $0.86(\mathrm{p}=0.01)$ and and interobserver agreement for $\mathrm{MCl}$ was $0.82(\mathrm{p}=0.05)$, according to the Kappa test

\section{Logistic regression results}

Adjusted odds ratio analysis showed no statistical significant results in the evaluation of horizontal, vertical and $\mathrm{MCl}$ bone loss between the two groups, as detailed in Table 2.

Logistic regression results showed no statistical significance for the variable "In the present study, we evaluated whether patients with type 2 diabetes and osteoporosis do not have an increased risk of periodontal disease (horizontal and vertical bone loss) when compared to diabetic patients without osteoporosis. All variables evaluated showed lack of significance with $p$ values higher than 0.05 .

\section{Discussion}

In the present study, we evaluated whether patients with type 2 diabetes and osteoporosis do not have an increased risk of periodontal disease (horizontal and vertical bone loss) when compared to diabetic patients without osteoporosis.

It is well known that there is a close relationship between the periodontal disease and diabetes. It is widely accepted in literature that periodontal disease, which leads to damage to connective tissue and alveolar bone, has greater prevalence and severity among diabetic patients when compared to non-diabetic patients ${ }^{13}$. Similarly, periodontitis has been referred to be strongly associated with osteoporosis ${ }^{14,15}$.

Diabetes interferes with the bone structure, and the disease is associated with an increased risk of fractures due to bone strength reduction, deteriorating "bone quality" instead of BMD1. Type 2 diabetic patients normally exhibit normal or higher BMD ${ }^{16}$. However, in recent studies, diabetes was associated with significant bone cortical porosity and trabecular defects, resulting from damaged osteoblast bone formation ${ }^{2}$. Also, hyperglycemia from decompensated diabetes increases activity and osteoblast proliferation and insulin resistance deteriorates osteoblast function, leading to osteoblastic apoptosis ${ }^{2}$. 
Periodontitis and osteoporosis are frequent diseases in older population, and the association between osteoporosis and periodontitis is discussed by many studies ${ }^{4}$. Periodontal disease bacterial infection has a well-established etiology; notwithstanding that, periodontitis as a result of osteoporosis possesses a probable important factor in the susceptibility to alveolar bone $\operatorname{loss}^{13}$. In a recent study with a large sample population studied ${ }^{4}$, researchers confirmed that osteoporotic patients have an increased risk of periodontitis; same conclusions were reported by other investigators 17,18 .

Tezal et al. ${ }^{17}$ hypothesized that systemic bony loss may contribute to periodontitis through four paths: 1) Alveolar bone is absorbed faster in patients with systemic bone loss compared to patients without osteoporosis simply because there is less bone to start with; 2) Patients with systemic bone loss may response to periodontal disease with enhanced production of proinflammatory cytokines; 3) Genetic factors predisposing to osteoporosis may be the same as predisposing to periodontitis, leading to rapid periodontal reabsorption; and 4) Environmental factors may increase the risk for both osteoporosis and periodontitis in a common way.

As previously mentioned, not only diabetic patients present periodontitis with greater prevalence and severity than non-diabetic patients, but also osteoporotic patients may have an increased risk to periodontal disease development. We expected, therefore, that osteoporotic diabetic patients would have substantially more periodontal disease than non-osteoporotic patients, but the present study could not demonstrate this association.

Furthermore, it wasn't observed an association between osteoporosis and low $\mathrm{MCl}$ in this study, although $\mathrm{MCl}$ is widely accepted to have an inverse correlation with T-scores among different populations $6,19,20$ and type 2 diabetes patients ${ }^{16}$. $\mathrm{MCl}$ classification reflects the systemic BMD; its well known that low BMD can result in alterations to mandibular shape, leading to loss of integrity of the inferior mandibular cortex ${ }^{21}$.

Many factors may have contributed to this negative association. First, the small sample size. Also, the lack of information about how long osteoporosis was diagnosed or how effective was glycemic control in type 2 diabetes patients. These factors may change the rate and severity of bone loss.

We should consider methodological limitations to this study. First, its retrospective nature, small convenience sample size and the lack of a control group. Additionally, ideally periodontal disease should be assessed by clinical examination and periapical radiographs. The present study evaluated periodontal disease by panoramic radiographs, which, although it is a useful examination to screen patients at periodontal disease risk, is not the most recommended examination in Periodontology. Hence, further larger sample prospective studies are recommended.

We haven't found in the literature published papers with a protocol similar to the present investigation, analyzing diabetic, periodontal and osteoporotic patients using panoramic radiographs and forearm DXA. Thus, this methodology will be valuable for future investigations on this topic. 
Within the limitations of the present study, we may conclude that there is no difference between the risk of periodontal disease development among osteoporotic and non-osteoporotic type 2 diabetic patients.

\section{Conflict of interest}

The authors state that they have no conflict of interest.

\section{References}

1. Saito M, Kida Y, Kato S, Marumo K. Diabetes, collagen, and bone quality. Curr Osteoporos Rep. 2014 Jun;12(2):181-8. doi: 10.1007/s11914-014-0202-7.

2. Wongdee K. Update on type 2 diabetes-related osteoporosis. World J Diabetes. 2015 Jun 10;6(5):673-8. doi: 10.4239/wjd.v6.i5.673

3. Irani FC, Wassall RR, Preshaw PM. Impact of periodontal status on oral health-related quality of life in patients with and without type 2 diabetes. J Dent. 2015 May;43(5):506-11. doi: 10.1016/j.jdent.2015.03.001.

4. Chang W-P, Chang W-C, Wu M-S, Pai J-T, Guo Y-C, Chen K-C, et al. Population-Based 5-Year Follow-Up Study in Taiwan of Osteoporosis and Risk of Periodontitis. J Periodontol. 2014 Mar;85(3):e24-30. doi: $10.1902 /$ jop. 2013.130256 .

5. Takeshita W, Vessoni Iwaki L, Da Silva M, Tonin R. Evaluation of diagnostic accuracy of conventional and digital periapical radiography, panoramic radiography, and cone-beam computed tomography in the assessment of alveolar bone loss. Contemp Clin Dent. 2014 Jul;5(3):318-23. doi: 10.4103/0976-237X.137930.

6. Munhoz L, Aoki EM, Cortes ARG, de Freitas CF, Arita ES. Osteoporotic alterations in a group of different ethnicity Brazilian postmenopausal women: An observational study. Gerodontology. 2018 Jun;35(2):101-109. doi: 10.1111/ger.12322.

7. American Dental Association. Dental radiographic examinations: recommendations for patient selection and limiting radioation exposure. FDA; revised 2012 [cited 2018 May 10]. Available from: https://www.ada.org/ /media/ADA/Member\%20Center/Flles/Dental_Radiographic_ Examinations_2012.ashx.

8. Du Bois AH, Kardachi B, Bartold PM. Is there a role for the use of volumetric cone beam computed tomography in periodontics? Aust Dent J. 2012 Mar;57 Suppl 1:103-8. doi: 10.1111/j.1834-7819.2011.01659.x.

9. Klemetti E, Kolmakov S, Kröger H. Pantomography in assessment of the osteoporosis risk group. Eur J Oral Sci. 1994 Feb;102(1):68-72.

10. Pimazoni Netto A, Andriolo A, Fraige Filho F, Tambascia M, Gomes MDB, Melo M, et al. [Update on glycated hemoglobin $(\mathrm{HbA} 1 \mathrm{C})$ for assessment of glycemic control and the diagnosis of diabetes: clinical and laboratory aspects]. Rv Bras Patol Med Lab. 2009 Feb;45(1):31-48. doi: 10.1590/S1676-24442009000100007. Portuguese.

11. Kanis JA. Assessment of fracture risk and its application to screening for postmenopausal osteoporosis: synopsis of a WHO report. WHO Study Group. Osteoporos Int. 1994 Nov;4(6):368-81.

12. Shapiro SS, Wilk MB. An analysis of variance test for normality (complete samples). Biometrika. 1965 Dec;52(3):591-611. doi: 10.2307/2333709.

13. Loe H. Periodontal disease: the sixth complicat diabetes mellitus. Diabetes Care. 1993 Jan;16:329-34 doi: $10.2337 /$ diacare.16.1.329. 
14. Passos JS, Vianna MIP, Gomes-Filho IS, Cruz SS, Barreto ML, Adan L, et al. Osteoporosis/osteopenia as an independent factor associated with periodontitis in postmenopausal women: A case-control study. Osteoporos Int. 2013 Apr;24(4):1275-83. doi: 10.1007/s00198-012-2130-7.

15. Kushi L, Folsom A, Prineas R, Mink P, Ying W, Bostick R. Postmenopausal women. N Engl J Med. 1996 May;334(18):1156-62

16. Munhoz L, Cortes A, Arita E. Assessment of osteoporotic alterations in type 2 diabetes: a retrospective study. Dentomaxillofac Radiol. 2017 Aug;46(6):20160414. doi: 10.1259/dmfr.20160414.

17. Tezal M, Wactawski-Wende J, Grossi SG, Ho AW, Dunford R, Genco RJ. The relationship between bone mineral density and periodontitis in postmenopausal women. J Periodontol. 2000 Sep;71(9):1492-8.

18. Payne JB, Reinhardt RA, Nummikoski PV, Patil KD. Longitudinal alveolar bone loss in postmenopausal osteoporotic/osteopenic women. Osteoporos Int. 1999;10(1):34-40.

19. Al-Dam A, Blake F, Atac A, Amling M, Blessmann M, Assaf A, et al. Mandibular cortical shape index in non-standardised panoramic radiographs for identifying patients with osteoporosis as defined by the German Osteology Organization. J Craniomaxillofac Surg. 2013 Oct;41(7):e165-9. doi: 10.1016/j.jcms.2012.11.044.

20. Dagistan S, Bilge OM. Comparison of antegonial index, mental index, panoramic mandibular index and mandibular cortical index values in the panoramic radiographs of normal males and male patients with osteoporosis. Dentomaxillofac Radiol. 2010 Jul;39(5):290-4. doi: $10.1259 / \mathrm{dmfr} / 46589325$.

21. Taguchi A, Ohtsuka M, Tsuda M, Nakamoto T, Kodama I, Inagaki K, et al. Risk of vertebral osteoporosis in post-menopausal women with alterations of the mandible. Dentomaxillofacial Radiol. 2007 Mar;36(3):143-8. doi: 10.1259/dmfr/50171930. 